\title{
Using Populations of Models to Navigate Big Data in Electrophysiology: Evaluation of Parameter Sensitivity of Action Potential Models
}

\author{
Carlos A. Ledezma ${ }^{1}$, Benjamin Kappler ${ }^{2}$, Veronique Meijborg ${ }^{3}$, Bas Boukens ${ }^{3}$, Marco Stijnen ${ }^{2}$, \\ PJ Tan ${ }^{1}$, Vanessa Díaz-Zuccarini ${ }^{1}$ \\ ${ }^{1}$ Department of Mechanical Engineering, University College London, London, United Kingdom \\ ${ }^{2}$ LifeTec Group, Eindhoven, Netherlands \\ ${ }^{3}$ Academic Medical Center, Amsterdam, Netherlands
}

\begin{abstract}
Experimentally-calibrated populations of models (ePoM) for cardiac electrophysiology can be used as a means to elucidate the cellular dynamics that lead to pathologies observed in organ-level measurements, while taking into account the variability inherent to living creatures. Notwithstanding, the results obtained through ePoM will depend on the capabilities of the template model, and not one model can accurately reproduce all pathologies. The objective of this work was to show how using different models, within an ePoM framework, can be advantageous when looking for the causes for a pathological behavior observed in experimental data. Populations of the ten Tusscher (2006) and the O'Hara-Rudy model were calibrated to activation-recovery intervals measured during an ex-vivo porcine heart experiment; a pathological reduction in ARI was observed as the experiment progressed in time. The ePoM approach predicted a reduction in calcium uptake via L-type channels, using the TPO6 model, and an increased potassium concentration in blood, using the ORd model, as the causes for the reduction in ARI; these findings were then confirmed by other experimental data. This approach can also accommodate different biomarkers or more mathematical models to further increase its predictive capabilities.
\end{abstract}

\section{Introduction}

Mathematical models for cardiac electrophysiology (EP) can be used to simulate the behavior of cardiomyocytes within a computerized environment. The most detailed models include descriptions for most of the ion channels present in the membrane, these channels allow the flow of ions in and out of the cell. Using these models researchers can study cellular action potentials, in-silico, without having to isolate individual myocytes for an invitro study; furthermore, these models can be used to simu- late both physiology and pathology in action potential genesis and propagation. Although numerous advances have been made in the field, and computational EP models have become incredibly detailed, there remain open questions that need to be addressed, particularly on how to choose a model that best suits the study at hand, about the choice of parameters and evaluation of parameter sensitivities, validation against reliable experimental data and in taking into account the role of inter-subject variability when studying both physiology and pathology [1].

Recently, 'experimentally-calibrated populations of models' (ePoM) have been used as a means to study the role of variability in EP models [2]. These populations are constructed by varying the maximal conductances and currents which are normally parameters of the EP models; this allows to investigate variability, which is not possible if assuming that these peak conductances and currents have a fixed value. Hence, a population of models is generated and by constraining the possible models of the population using experimental data one can hypothesize what are the conditions within the cellular membrane that could produce an observed physiological behavior. Previous works in populations of models have shown how this approach can be used to characterize abnormal behaviors in atrial and ventricular tissue $[3,4]$.

The caveat of using a template model to describe experimental observations is that the results are highly dependent on the mathematical formulation. Previous authors have made quantitative comparisons in the behavior of models for cardiac EP and found that they differ [5]. Other works have also shown quantitative differences when simulating pathologies with EP models [6]. The objective of this study was to investigate how to take advantage of the different sensitivities of the most commonly used mathematical models for cardiac EP to understand the cellular level pathologies that could lead to abnormal behaviors observed in measurements made on the surface of the heart. For this, two populations of different models were 
calibrated to the same experimental data and the results obtained using the different populations of models were compared in search for quantitative differences; then, the results were interpreted taking into account the limitations of each model to draw conclusions on the possible causes for the observed pathological behavior. This work provides a template for the analysis of experimental data, using populations of models, that could be applied on different datasets and using different models. Also, it shows the importance of using different computational models when analyzing experimental data, since the conclusions drawn from a single model may lead to an incomplete diagnose.

\section{Materials and Methods}

This work uses 'experimentally-calibrated populations of models' (ePoM) as a means to analyze the changes in activation-recovery intervals (ARI) observed during an $e x$ vivo porcine heart experiment, to be presented shortly. Two populations of models were generated, as explained by previous authors [4], by varying the otherwise fixed parameters of two widely used models for cardiac EP. The first population was created using the ten Tusscher-NobleNoble-Panfilov model (TP06) [7] as a template and the second one using the O'Hara-Rudy model (ORd) [8]. The TP06 model is the most widely used and studied model for ventricular myocytes, but it is does not adequately reproduce AP response to frequency changes and potassium current block; on the other hand, the ORd model is the most recent human ventricular model and was based on human data from over 100 undiseased hearts, but is incapable, in its original formulation, of reproducing the effects of hyperkalemia in tissue [9]. Observe that, even though the TP06 and the ORd are human models, they can be used to characterize porcine myocyte activity because the swine and human electrophysiology are similar in the underlying ionic dynamics; the inter-species differences in current magnitudes are accounted for in the generation of the populations of models.

The experimental data used in this study was acquired during a Physioheart experiment following the guidelines outlined in previous studies [10]. The performance of the porcine heart during the experiment was measured by means of the cardiac output. The porcine heart maintained a cardiac output of around $4 \mathrm{~L} / \mathrm{min}$ for over 2 hours of experiment, this time was called the working mode. After 150 minutes in working mode the cardiac output dropped to $2.7 \mathrm{~L} / \mathrm{min}$, this was called the experiment's 'end-point'. During the experiment the heart was paced at $600 \mathrm{~ms}$ cycle length. The electrical activity of the heart was assessed using left ventricular surface epicardial electrograms (EGM), acquired by means of a grid of 121 electrodes, from which activation-recovery intervals (ARI) were measured. Measurements were made at three representative times of the experiment: $90 \mathrm{~min}, 120 \mathrm{~min}$ and $150 \mathrm{~min}$ after the start of the working mode. The ARI is a surrogate measurement for action potential duration at $90 \%$ repolarization (APD90) [11], but it provides no further information on action potential shape; different action potential morphologies can have the same APD90, which means that two cells exhibiting the same duration can be under different physiological conditions, this is called inter-subject variability.

The aforementioned populations of models were used to monitor the temporal evolution of the physiological condition of the cellular membrane under each of the electrodes of the grid, while accounting for variability. One ePoM was formed by keeping only the models from a population that were in the range $\mu_{A R I} \pm \sigma_{A R I}$ of one channel, at one time point; where $\mu_{A R I}$ was the mean value of the ARIs measured on that channel at that time point and $\sigma_{A R I}$ was their standard deviation. So, an ePoM from one channel, at one time point, contained all the possible parameter combinations that could have lead to the ARI observed at that channel, at that time point. EPoMs were constructed for all channels, at all time points, and a 'pathological' ePoM was identified when significant differences in the statistical distributions of its parameters were found when comparing to a 'healthy' ePoM coming from the same channel. The stable cardiac output and homogeneous ARI, as will be shown in the results, allowed to identify the ePoMs at the beginning of the experiment as healthy. In line with previous ePoM studies, statistically significant differences were searched for using a Mann-Whitney-Wilcoxon U-Test [4]. The calibration process and statistical analysis was repeated with both the TP06 and the ORd populations if models. The results obtained by applying the same methodology, on the same data, with two different models are presented and discussed in the following sections.

\section{Results}

The summary of the data acquisitions can be observed in Table 1, where the measured ARIs are summarized, within their respective time points, by means of their mean $\left(\mu_{A R I}\right)$, standard deviation $\left(\sigma_{A R I}\right)$, maximum and minimum values. It can be observed that at the time of the first measurement the cardiac output was at a stable value of $3.9 \mathrm{~L} / \mathrm{min}$, which continued to be stable even 30 minutes after, as can be observed in the second time point; also, at the first time point the ARI were homogeneous along the membrane, as can be observed from the mean and standard deviation of their values. However, even when the cardiac output was stable in the second time point, the ARIs were shortening on the membrane, as can be observed from the larger standard deviation and lower minimum ARI. Sixty minutes after the first time point there was a sudden decrease in ARI, accompanied by a significant decrease in cardiac output, this marked the 'end point' of the experi- 
ment.

The causes for the aforementioned reduction in activation-recovery intervals were investigated by means of the ePoM methodology described in the previous section. The results of applying the methodology, with each of the mathematical models, can be observed in Table 2. This table shows the number of channels that displayed a statistically significant difference in a given parameter when comparing the populations calibrated at time points 2 and 3 to their counterparts at time point 1 . Results using the TP06 model indicate that there was an increasing amount of sites of the membrane with an impaired L-type calcium conductance, this number was close to half of the channels at time point 2 and increased to $70 \%$ of the channels at time point 3; further analyses showed that this impairment was a reduced conductance. On the other hand, the methodology applied using the ORd model pointed at an increase in the delayed rapid rectifier potassium conductance as the main cause for the reduction in ARIs observed during the experiment; using the ORd PoM half of the channels displayed a statistically significant increase in $G_{K r}$ at time point 2 and 86 channels show a further increase at time point 3 .

\section{Discussion}

From the results presented in Table 1 it is possible to conclude that the cardiac failure observed during the experiment was accompanied by a pathological reduction in the activation-recovery intervals over the surface of the heart. This abnormal electrical behavior was observable well before the reduction in cardiac output was evident, which means that pathology can be identified at an earlier stage if the cellular, rather than the organ, behavior is monitored. Notwithstanding, there are many possible causes for the reduction in action potential duration and given that the direct measurement of ion dynamics across the membrane is extremely difficult, a computational approach is justified as a means to elucidate the underlying dynamics that produced the observed reduction in ARI. Furthermore, given that models have different parameter sensitivities, it is interesting to use several formulations to investigate all the possible causes for the reduction in APD90.

The results obtained using the TP06 model seem to point that a reduction in L-type calcium conductance was the main responsible for the observed reduction in cardiac output. This seems consistent with other acquired experimental data, which showed reduction in myocyte contractility and increased calcium levels in blood as the experiment progressed in time; this points to the TP06 model being able to identify the underlying mechanisms that caused cardiac failure. On the other hand, the results obtained using the ORd model point at an increase in $G_{K r}$ as the most likely cause for the reduction in ARI; note that this is not in contradiction with the TP06 prediction, because the TP06 does not react properly to potassium current changes. Nevertheless, blood analyses also showed that the potassium concentration in blood was abnormally high throughout the experiment, and the ORd model was solved assuming a normal concentration in blood. $I_{K r}$ is directly proportional to the extracellular potassium concentration; hence, it is possible that the increase observed in $G_{K r}$ was a reflection of the high potassium concentration as opposed to a channel over-expression. This is further supported by the fact that a number of channels also showed elevated $G_{K r}$ when using the TP06 model, something that wouldn't be possible if the ARI reduction was due just to rapid delayed rectifier potassium channel overexpression.

It is evident how meaningful conclusions can be obtained using a single dataset and taking advantage of the different sensitivities of cardiac EP models. The calibration of populations of different models allowed to investigate different possible causes for the observed pathological behavior, and the use of other experimental data allowed to confirm the hypotheses made by the analysis of ePoMs. It is worthwhile to observe that there is little information contained in the ARI, it is a surrogate measurement for a single AP biomarker, but the use of different mathematical models in concert with statistical methods allowed to reach relevant conclusions from this somewhat limited dataset. Finally, one can observe that the approach taken here can easily be adapted to accommodate more mathematical formulations and even more action potential measurements, making it an ideal template for the analysis of cardiac EP phenomena through mathematical models.

\section{Conclusions}

A method based on 'experimentally-calibrated populations of models' has been presented, and its ability to draw conclusions from limited datasets has been proven. Populations of the TP06 and ORd models were generated and calibrated to activation-recovery intervals measured from epicardial electrograms acquired during a Physioheart experiment. The statistical analysis of these populations allowed to infer what was, most likely, causing the reduction in ARI observed during the experiment. Calibrated populations of the TP06 model predicted a reduction in Ltype calcium conductance as the cause for the shortened ARIs, whereas the ORd populations pointed at an increase in extra-cellular potassium concentration. Indeed, other experimental data were in agreement with the conclusions reached by both models, thus showing that a single mathematical formulation is not enough to decide what are the causes for pathology when a single biomarker is used. It is expected that the inclusion of more biomarkers and mathematical formulations will produce better predictions when this methodology is applied. 
Table 1. Data collected at each time point. In parentheses the minutes after the beginning of the working mode.

\begin{tabular}{lcccccc}
\hline \hline Time point & Duration & $\mathrm{CO}$ & $\mu_{A R I}$ & $\sigma_{A R I}$ & $\max$ & $\min$ \\
& & & & & & \\
\hline $1(90 \mathrm{~min})$ & $92 \mathrm{~s}$ & $3.8 \mathrm{~L} / \mathrm{min}$ & $258.5 \mathrm{~ms}$ & $6.6 \mathrm{~ms}$ & $276.4 \mathrm{~ms}$ & $219.2 \mathrm{~ms}$ \\
$2(120 \mathrm{~min})$ & $77 \mathrm{~s}$ & $3.7 \mathrm{~L} / \mathrm{min}$ & $252.8 \mathrm{~ms}$ & $12.5 \mathrm{~ms}$ & $295.9 \mathrm{~ms}$ & $206.1 \mathrm{~ms}$ \\
$3(150 \mathrm{~min})$ & $44 \mathrm{~s}$ & $2.7 \mathrm{~L} / \mathrm{min}$ & $235.1 \mathrm{~ms}$ & $38.4 \mathrm{~ms}$ & $285.6 \mathrm{~ms}$ & $100.1 \mathrm{~ms}$ \\
\hline \hline
\end{tabular}

Table 2. Number of channels showing a statistically significant difference in the parameters of their ePoMs when comparing time points 2 and 3 to time point 1.

\begin{tabular}{cccccc}
\hline \hline \multicolumn{3}{c}{ TP06 } & \multicolumn{4}{c}{ ORd } \\
\hline Parameter & $1-2$ & $1-3$ & Parameter & $1-2$ & $1-3$ \\
\hline$G_{N a}$ & 0 & 0 & $G_{N a}$ & 0 & 0 \\
$G_{t o}$ & 0 & 0 & $G_{t o}$ & 1 & 7 \\
$G_{K s}$ & 6 & 11 & $G_{K s}$ & 0 & 0 \\
$G_{K r}$ & 11 & 14 & $G_{K r}$ & 65 & 86 \\
$G_{k 1}$ & 0 & 0 & $G_{k 1}$ & 0 & 4 \\
$P_{N a K}$ & 2 & 2 & $P_{N a K}$ & 7 & 2 \\
$G_{C a L}$ & 61 & 85 & $P_{C a}$ & 0 & 4 \\
$k_{N a C a}$ & 0 & 0 & $G_{n c x}$ & 0 & 1 \\
$G_{p C a}$ & 3 & 5 & $G_{p C a}$ & 0 & 0 \\
$G_{p K}$ & 0 & 0 & $G_{K b}$ & 0 & 0 \\
$G_{b N a}$ & 0 & 0 & $P_{N a b}$ & 9 & 0 \\
$G_{b C a}$ & 0 & 0 & $P_{C a b}$ & 0 & 1 \\
& & & $G_{N a L}$ & 0 & 1 \\
& & & $P_{j r e l}$ & 0 & 0 \\
& & & $P_{j u p}$ & 0 & 0 \\
\hline \hline
\end{tabular}

\section{Acknowledgements}

This project has received funding from the European Union's Horizon 2020 research and innovation programme under the Marie Sklodowska-Curie grant agreement No 642612, VPH-CaSE (www.vph-case.eu)

\section{References}

[1] Clayton R, Bernus O, Cherry E, Dierckx H, Fenton F, Mirabella L, Panfilov A, Sachse FB, Seemann G, Zhang H. Models of cardiac tissue electrophysiology: progress, challenges and open questions. Progress in biophysics and molecular biology 2011;104(1):22-48.

[2] Muszkiewicz A, Britton OJ, Gemmell P, Passini E, Sánchez C, Zhou X, Carusi A, Quinn TA, Burrage K, BuenoOrovio A, et al. Variability in cardiac electrophysiology: Using experimentally-calibrated populations of models to move beyond the single virtual physiological human paradigm. Progress in biophysics and molecular biology 2016;120(1):115-127.

[3] Muszkiewicz A, Bueno-Orovio A, Liu X, Casadei B, Ro- driguez B. Constructing human atrial electrophysiological models mimicking a patient-specific cell group. In Computing in Cardiology Conference (CinC), 2014. IEEE, 2014; 761-764.

[4] Zhou X, Bueno-Orovio A, Orini M, Hanson B, Hayward M, Taggart P, Lambiase PD, Burrage K, Rodriguez B. In vivo and in silico investigation into mechanisms of frequency dependence of repolarization alternans in human ventricular cardiomyocytes. Circulation research 2016;118(2):266278.

[5] Elshrif MM, Cherry EM. A quantitative comparison of the behavior of human ventricular cardiac electrophysiology models in tissue. PloS one 2014;9(1):e84401.

[6] Zimik S, Vandersickel N, Nayak AR, Panfilov AV, Pandit R. A comparative study of early afterdepolarization-mediated fibrillation in two mathematical models for human ventricular cells. PloS one 2015;10(6):e0130632.

[7] ten Tusscher KH, Panfilov AV. Alternans and spiral breakup in a human ventricular tissue model. American Journal of Physiology Heart and Circulatory Physiology 2006; 291(3):H1088-H1100.

[8] O'Hara T, Virág L, Varró A, Rudy Y. Simulation of the undiseased human cardiac ventricular action potential: model formulation and experimental validation. PloS Computational Biology 2011;

[9] Dutta S, Mincholé A, Quinn TA, Rodriguez B. Electrophysiological properties of computational human ventricular cell action potential models under acute ischemic conditions. Progress in Biophysics and Molecular Biology 2017;

[10] de Hart J, de Weger A, van Tuijl S, Stijnen J, van den Broek CN, Rutten M, de Mol BA. An ex vivo platform to simulate cardiac physiology: a new dimension for therapy development and assessment. The International journal of artificial organs 2011;34(6):495-505.

[11] Potse M, Vinet A, Opthof T, Coronel R. Validation of a simple model for the morphology of the $\mathrm{T}$ wave in unipolar electrograms. American Journal of Physiology Heart and Circulatory Physiology 2009;297(2):H792-H801.

Address for correspondence:

Name: Vanessa Díaz-Zuccarini

Address: Department of Mechanical Engineering, UCL, London, WC1E 7JE

E-mail address: v.diaz@ucl.ac.uk 